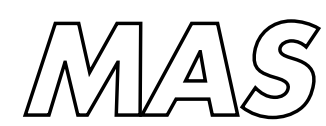

Modelling, Analysis and Simulation

Modelling, Analysis and Simulation
MAS A Pressure-Invariant and Conservative Method for
Two-Fluid Flows

H. van Brummelen, B. Koren

Report MAS-E0323 December 16, 2003 
CWI is the National Research Institute for Mathematics and Computer Science. It is sponsored by the Netherlands Organization for Scientific Research (NWO).

CWI is a founding member of ERCIM, the European Research Consortium for Informatics and Mathematics.

CWI's research has a theme-oriented structure and is grouped into four clusters. Listed below are the names of the clusters and in parentheses their acronyms.

Probability, Networks and Algorithms (PNA)

Software Engineering (SEN)

Modelling, Analysis and Simulation (MAS)

Information Systems (INS)

Copyright (C) 2003, Stichting Centrum voor Wiskunde en Informatica

P.O. Box 94079, 1090 GB Amsterdam (NL)

Kruislaan 413, 1098 SJ Amsterdam (NL)

Telephone +31205929333

Telefax +31205924199

ISSN 1386-3703 


\title{
A Pressure-Invariant and Conservative Method for Two-Fluid Flows
}

\begin{abstract}
Discretizations of two-fluid flow problems in conservative formulation generally exhibit pressure oscillations. In this work we show that these pressure oscillations are induced by the loss of a pressure-invariance property under discretization, and we introduce a non-oscillatory conservative method for barotropic two-fluid flows. The conservative formulation renders the two-fluid flow problem suitable to treatment by a Godunov-type method. We present a modified Osher scheme for the two-fluid flow problem. Numerical results are presented for a translatinginterface test case and a shock/interface-collision test case.
\end{abstract}

2000 Mathematics Subject Classification: 76-xx

Keywords and Phrases: barotropic two-fluid flows; capturing method 


\title{
A PRESSURE-INVARIANT AND CONSERVATIVE METHOD FOR TWO-FLUID FLOWS
}

\author{
Harald van Brummelen \\ CWI \\ P.O. Box 94079, 1090 GB Amsterdam, The Netherlands \\ Currently: \\ Delft University of Technology, Faculty of Aerospace Engineering \\ P.O. Box 5058, 2600 GB Delft, The Netherlands \\ e-mail: e.h.vanbrummelen@1r.tudelft.nl \\ Barry Koren \\ CWI and Delft University of Technology, Faculty of Aerospace Engineering \\ P.O. Box 5058, 2600 GB Delft, The Netherlands \\ e-mail: barry.koren@cwi.nl \\ senior member AIAA
}

\begin{abstract}
$\underline{\text { ABSTRACT }}$
Discretizations of two-fluid flow problems in conservative formulation generally exhibit pressure oscillations. In this work we show that these pressure oscillations are induced by the loss of a pressure-invariance property under discretization, and we introduce a non-oscillatory conservative method for barotropic two-fluid flows. The conservative formulation renders the two-fluid flow problem suitable to treatment by a Godunov-type method. We present a modified Osher scheme for the two-fluid flow problem. Numerical results are presented for a translating-interface test case and a shock/interface-collision test case.
\end{abstract}

\section{INTRODUCTION}

Flows of two immiscible contiguous fluids occur in a multitude of physical sciences and engineering applications, e.g., water underlying air in ship hydrodynamics, gaseous bubbles in cavitating liquids and fumes in petrolea. Such two-fluids can be construed as a single medium sustaining a discontinuity at the interface. In the absence of viscosity, a two-fluid flow is then described by a system of hyperbolic conservation laws. The numerical treatment of twofluid flows as a system of hyperbolic conservation laws is referred to as interface capturing. For examples of interface capturing see, for instance, Refs. ${ }^{6,16,20}$.

A common objection to conservative interface capturing is the occurrence of so-called pressure oscillations. These pressure oscillations expose the loss of certain invariance properties of the continuum problem under discretization. Several correctives have been proposed to avoid pressure oscillations, e.g., (locally) non-conservative discretization methods $1,14,15,23$, correction methods ${ }^{13}$ and the ghostfluid method ${ }^{7}$. For an overview of these correctives, and of their merits and deficiencies, see ${ }^{2}$ and, for homentropic flows, Ref. ${ }^{17}$. A characteristic of these methods is that at the interface the conservative formulation is abandoned. Hence, these methods are generally non-conservative. Recently, enhancements of the ghost-fluid method have been proposed, which retain conservation; see Refs. ${ }^{8,21}$. However, the interface treatment of these methods is not trivial and further investigation is warranted.

It is commonly assumed that the loss of the aforementioned invariance properties is inherent to any conservative formulation; see, e.g., Refs. ${ }^{2,24}$. However, since the invariance properties are intrinsic to the continuum equations, irrespective of their form, we conjecture that it is possible to devise conservative numerical schemes that inherit the necessary invariance properties.

The interface-capturing approach requires that the employed numerical techniques remain robust and accurate in the presence of discontinuities. If one adheres to the conservative form of the equations, then Godunov-type schemes ${ }^{9}$ are particularly useful in these circumstances.

The present work considers the interface-capturing approach to solving two-fluid flow problems. We investigate the pressure oscillations that are commonly incurred by discrete approximations of two-fluid flow problems, and we present a non-oscillatory, conservative Godunov-type method for barotropic fluids. Moreover, we set up a modified Osher-type flux-difference splitting scheme for the approximate solution of the two-fluid Riemann problems. The novelty of our method is its pressure invariance in combination with a formulation of the two-fluid flow problem as a system of hyperbolic conservation laws. It is generally accepted that methods based on such a formulation necessarily exhibit pressure oscillations; our results refute this.

\section{TWO-FLUID FLOWS}

The basic notion underlying the interface capturing method, is that a flow of two contiguous, inviscid com- 
pressible fluids can be construed as a flow of a single medium sustaining a discontinuity at the interface. In this section we derive the two-fluid Euler equations from the Euler equations for the separate fluids and the interface conditions.

\section{CONSERVATION LAWS}

We consider flows of two contiguous inviscid compressible fluids. For convenience, we arbitrarily designate one of the fluids as the primary fluid and the other as the secondary fluid. For our purposes, it suffices to consider a single spatial dimension. We refer to the corresponding spatial coordinate as $x$ and to the temporal coordinate as $t$. The fluids occupy an open bounded space/time domain $\Omega \subset\left\{(x, t) \in \mathbb{R}^{2}\right\}$, which is the union of the disjoint open sets $\Omega_{\mathrm{p}}$ and $\Omega_{\mathrm{s}}$, containing the primary and secondary fluid, respectively, and the interface $\Gamma:=\overline{\Omega_{\mathrm{p}}} \cap \overline{\Omega_{\mathrm{s}}}$ (the overbar denoting closure); see Figure 1.

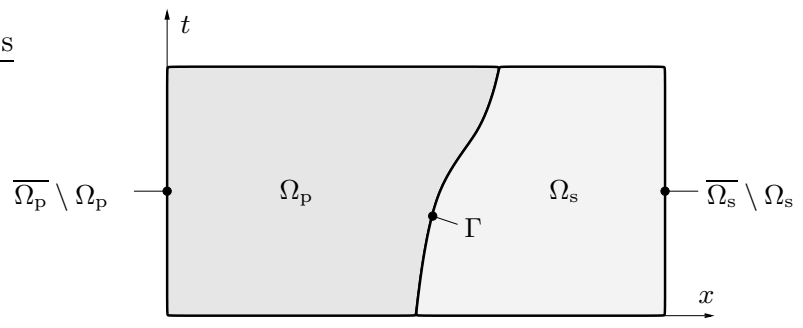

Figure 1: The space/time domain $\Omega:=\Omega_{\mathrm{p}} \cup \Omega_{\mathrm{s}} \cup \Gamma$.

In both fluids the flow is characterized by the state variables $\rho: \Omega \mapsto \mathbb{R}_{+}$and $v: \Omega \mapsto \mathbb{R}$, representing density and velocity, respectively. To facilitate the presentation of the governing equations, we introduce the notation:

$$
\mathbf{q}:=\left(\begin{array}{c}
\rho \\
\rho v
\end{array}\right), \quad \text { and } \quad \mathbf{f}(\mathbf{q}):=\left(\begin{array}{c}
q_{2} \\
q_{2}^{2} / q_{1}+p
\end{array}\right)
$$

where $p$ refers to the pressure. Eq. (1) must be furnished with equations of state for the primary and secondary fluid. Under the assumption that the fluids are barotropic (see, e.g., Ref. ${ }^{29}$ ), these equations of state have the form $p:=p_{\mathrm{p}}(\rho)$ and $p:=p_{\mathrm{s}}(\rho)$. In a proper functional setting, conservation of mass and momentum in the fluids is expressed by the variational statement

$\int_{\Omega} \mathbf{w}_{t} \cdot \mathbf{q}+\mathbf{w}_{x} \cdot \mathbf{f}(\mathbf{q}) d x d t=0, \quad \forall \mathbf{w} \in\left[C_{0}^{\infty}\left(\Omega_{\mathrm{p}} \cup \Omega_{\mathrm{s}}\right)\right]^{2}$,

where $C_{0}^{\infty}(G)$ denotes the space of functions that have continuous partial derivatives of all orders $k=0,1,2, \ldots$. and that have compact support in $G$.

\section{INTERFACE CONDITIONS}

To present the interface conditions for the two-fluid flow, we define

$$
(x, t)^{ \pm}:=\lim _{\epsilon \downarrow 0}(x \pm \epsilon, t), \quad(x, t) \in \Gamma,
$$

i.e., $(x, t)^{-}$and $(x, t)^{+}$are at the interface in the primary and secondary fluid, respectively. The interface conditions for the two-fluid flow prescribe that the velocity and pressure are continuous across the interface. In particular,

$$
\begin{array}{ll}
\left.v\right|_{(x, t)^{-}} ^{(x, t)^{+}}=0, & (x, t) \in \Gamma, \\
\left.p\right|_{(x, t)^{-}} ^{(x,)^{+}}=0, & (x, t) \in \Gamma .
\end{array}
$$

Eq. (4b) is referred to as the dynamic condition. Furthermore, the interface motion must comply with a kinematic condition. To express this kinematic condition, we identify the interface by a level set:

$$
\Gamma:=\{(x, t) \in \Omega: \theta(x, t)=0\},
$$

with $\theta \in C^{\infty}(\Omega)$ a suitably chosen function. We assume that $\theta\left(\Omega_{\mathrm{p}}\right)>0$ and $\theta\left(\Omega_{\mathrm{s}}\right)<0$. The kinematic interface condition is stated:

$$
\theta_{t}+v \theta_{x}=0, \quad(x, t) \in \Omega .
$$

Eq. (4c) implies that the interface moves with the local flow velocity and thus ensures immiscibility. Recall that the velocity at the interface is uniquely defined by virtue of (4a).

\section{TWo-Fluid Euler EQUATIONS}

To formulate the two-fluid Euler equations, it is important to note that the interface conditions (4) imply that the Rankine-Hugoniot condition for discontinuities in hyperbolic systems (see, for instance, Ref. ${ }^{27}$ ) is satisfied at the interface:

$$
\begin{array}{r}
s\left(\mathbf{q}(x, t)^{+}-\mathbf{q}(x, t)^{-}\right)=\mathbf{f}\left(\mathbf{q}(x, t)^{+}\right)-\mathbf{f}\left(\mathbf{q}(x, t)^{-}\right), \\
(x, t) \in \Gamma,
\end{array}
$$

with $s$ the shock speed. In particular, for the interface, $s=v(x, t)$ for $(x, t) \in \Gamma$. The variational statement (2) subject to (5) is equivalent to

$$
\int_{\Omega} \mathbf{w}_{t} \cdot \mathbf{q}+\mathbf{w}_{x} \cdot \mathbf{f}(\mathbf{q}) d x d t=0, \quad \forall \mathbf{w} \in\left[C_{0}^{\infty}(\Omega)\right]^{2} .
$$

Note that the functions $\mathbf{w}$ in (6) can have support across the interface, in contrast to (2). The equivalence is founded on the classical principle that a piecewise continuous solution is a valid weak solution if and only if it satisfies the Rankine-Hugoniot condition at discontinuities.

To obtain a conservative formulation of the two-fluid Euler equations, we must replace the nonconservative, advective form of the kinematic condition (4c) by a conservative equivalent. Under the conditions imposed by (6), an appropriate replacement for $(4 \mathrm{c})$ is:

$$
\int_{\Omega} \lambda_{t} \rho g(\theta)+\lambda_{x} \rho g(\theta) v d x d t=0, \quad \forall \lambda \in C_{0}^{\infty}(\Omega),
$$


with $\theta \mapsto g(\theta)$ a strictly monotone map with the property that for all $\lambda \in C_{0}^{\infty}(\Omega)$ and for all admissible $(\rho, \rho v)$ there exists a $w \in C_{0}^{\infty}(\Omega)$ such that

$$
\begin{array}{r}
\int_{\Omega} w_{t} \rho+w_{x} \rho v d x d t=\int_{\Omega}\left(\lambda_{t} g(\theta)+\lambda g^{\prime}(\theta) \theta_{t}\right) \rho+ \\
\left(\lambda_{x} g(\theta)+\lambda g^{\prime}(\theta) \theta_{x}\right) \rho v d x d t . \quad(7 \mathrm{~b})
\end{array}
$$

If $g$ is a $C^{\infty}$ map then $\lambda g(\theta) \in C^{\infty}(\Omega)$ and the identity (7b) follows by setting $w=\lambda g(\theta)$ and invoking partial differentiation. However, even if $g$ is less regular, e.g., piecewise $C^{\infty}$, then the condition can still be satisfied if the derivatives are understood in a generalized sense. To establish that (6) and (7a) imply (4c), we note that by (6) and (7b)

$$
\begin{aligned}
& \int_{\Omega} \lambda_{t} \rho g(\theta)+\lambda_{x} \rho g(\theta) v d x d t+ \\
& \int_{\Omega} \lambda_{x} \rho g^{\prime}(\theta)\left(\theta_{t}+v \theta_{x}\right) d x d t=0, \quad \forall \lambda \in C_{0}^{\infty}(\Omega) .
\end{aligned}
$$

By virtue of (7a), the integrals in (8) must vanish separately. Therefore, Eq. (6) and (7a) imply (4c) weakly.

To conclude the setup of the two-fluid Euler equations, we note that the interface conditions (4) are identical to the continuity conditions for contact discontinuities; see, e.g., Refs. ${ }^{27,29}$. Therefore, the two-fluid flow problem can be condensed into the variational statement

$$
\int_{\Omega} \mathbf{w}_{t} \cdot \mathbf{q}+\mathbf{w}_{x} \cdot \mathbf{f}(\mathbf{q}) d x d t=0, \quad \forall \mathbf{w} \in\left[C_{0}^{\infty}(\Omega)\right]^{3},
$$

where

$$
\mathbf{q}:=\left(\begin{array}{c}
\rho \\
\rho v \\
\rho g(\theta)
\end{array}\right), \quad \text { and } \quad \mathbf{f}(\mathbf{q}):=\left(\begin{array}{c}
q_{2} \\
q_{2}^{2} / q_{1}+p \\
q_{3} q_{2} / q_{1}
\end{array}\right)
$$

with the provision that $\theta$ can only change sign across a contact discontinuity, i.e., that the interface coincides with a contact discontinuity. In the following we shall show that (9) indeed complies with the latter requirement.

Eq. (9) must be equipped with a compound equation of state of the form $p:=p(\rho, \theta)$ with the property:

$$
p(\rho, \theta):= \begin{cases}p_{\mathrm{p}}(\rho) & \text { if } \theta>0, \\ p_{\mathrm{s}}(\rho) & \text { if } \theta<0 .\end{cases}
$$

One may note that in (9)-(10), $\theta$ only acts as an intermediary between $g$ and $p$. Therefore, $\theta$ does not have to appear explicitly in the formulation.

\section{Pressure Oscillations}

A common objection to interface capturing is the occurrence of pressure oscillations. These pressure oscillations expose the loss of the pressure-invariance property of the continuum problem under discretization. Below, we exemplify the pressure oscillations and we derive a pressureinvariance condition for discrete approximations to twofluid flow problems. Furthermore, we construct a nonoscillatory conservative discretization for barotropic twofluid flows.

\section{EXEMPLIFICATION}

The ensuing exemplification has appeared in similar form in, e.g., Refs. ${ }^{2,17,24}$ and is merely included here for completeness.

To illustrate the pressure oscillations that are generally incurred by conservative discretizations of two-fluid flow problems, we consider (9) on $\Omega:=\mathcal{L} \times] 0, \infty$, with $\mathcal{L}$ an open bounded subset of $\mathbb{R}$. We assign $g$ as the primary volume fraction. In particular, this implies

$$
g(\theta):= \begin{cases}1 & \text { if } \theta>0 \\ 0 & \text { otherwise }\end{cases}
$$

The compound equation of state is specified accordingly as

$$
\rho(p, \theta)=g(\theta) \rho_{\mathrm{p}}(p)+(1-g(\theta)) \rho_{\mathrm{s}}(p),
$$

with $\rho_{\mathrm{p}}(p)$ and $\rho_{\mathrm{s}}(p)$ the equations of state for the primary and secondary fluid. In fact, (12) provides a definition of the volume fraction in terms of $p$ and $\rho$. We allude to the fact that $\theta$ can be removed from the formulation and we suppress the dependence of $g$ on $\theta$ below.

The spatial interval $\mathcal{L}$ is subdivided into open intervals $\left.\mathcal{L}_{j}:=\right] x_{j}, x_{j+1}[$ with $j=1, \ldots, n$ and (9)-(12) is supplemented with the initial conditions

$$
\begin{aligned}
\rho(x, 0)=\rho_{j}^{0}, \quad & v(x, 0)=V, \quad g(x, 0)=g_{j}^{0}, \\
& x \in] x_{j}, x_{j+1}[, \quad j=1, \ldots, n,
\end{aligned}
$$

with $V$ an arbitrary positive constant and $\rho_{j}^{0}$ and $g_{j}^{0}$ constants such that

$$
\rho_{j}^{0}=g_{j}^{0} \rho_{\mathrm{p}}(P)+\left(1-g_{j}^{0}\right) \rho_{\mathrm{s}}(P),
$$

for some constant $P$. The equations (9)-(13) represent a two-fluid flow in which the velocity $v$ is uniform and in which the density $\rho$ and the primary volume fraction $g$ are such that the pressure $p$ is uniform as well.

The obvious solution to (9)-(13) is given by

$$
\mathbf{q}(x, t)=\mathbf{q}(x-V t, 0) .
$$

The pressure $p(x, t)$ corresponding to (14) follows from the compound equation of state:

$$
\rho(x, t)=g(x, t) \rho_{\mathrm{p}}(p(x, t))+(1-g(x, t)) \rho_{\mathrm{s}}(p(x, t)) .
$$

By (14)-(15),

$$
\begin{aligned}
\rho(x-V t, 0)= & g(x-V t, 0) \rho_{\mathrm{p}}(p(x, t))+ \\
& (1-g(x-V t, 0)) \rho_{\mathrm{s}}(p(x, t)),
\end{aligned}
$$


and it follows that $p(x, t)=P$. In conclusion, if the initial velocity and pressure are uniform, then the pressure is invariant under (9).

To illustrate the loss of the pressure-invariance property, we consider the discretization of (9)-(13) on the grid $\left\{\left(x_{j}, t_{k}\right): j=1, \ldots, n, k=1,2, \ldots\right\}\left(t_{0}=0\right.$ and $\left.t_{k}<t_{k+1}\right)$ by means of the discontinuous Galerkin finite element method with piecewise constants:

$$
\frac{\mathbf{q}_{j}^{k+1}-\mathbf{q}_{j}^{k}}{t_{k+1}-t_{k}}+\frac{\mathbf{f}\left(\mathbf{q}_{j}^{k}, \mathbf{q}_{j+1}^{k}\right)-\mathbf{f}\left(\mathbf{q}_{j-1}^{k}, \mathbf{q}_{j}^{k}\right)}{x_{j+1}-x_{j}}=0 .
$$

This discretization is a first-order forward Euler finitevolume discretization. We specify the initial conditions $\mathbf{q}_{j}^{0}=\left(\rho_{j}^{0}, \rho_{j}^{0} V, \rho_{j}^{0} g_{j}^{0}\right)^{T}$, in conformity with (13). In (17), $\mathbf{f}\left(\mathbf{q}_{j}^{k}, \mathbf{q}_{j+1}^{k}\right)$ refers to the numerical flux (see, e.g., Ref. ${ }^{11}$ ) between the elements $\mathcal{L}_{j}$ and $\mathcal{L}_{j+1}$. The grid function $\mathbf{q}_{j}^{k}$ is a piecewise constant approximation to $\mathbf{q}\left(x, t_{k}\right)$ according to (14) in the interval $\mathcal{L}_{j}$.

The states $\mathbf{q}_{j}^{0}$ and $\mathbf{q}_{j+1}^{0}(j=1, \ldots, n-1)$ are connected by a contact discontinuity with velocity $V$. The corresponding Godunov flux becomes:

$$
\mathbf{f}\left(\mathbf{q}_{j}^{0}, \mathbf{q}_{j+1}^{0}\right)=V\left(\begin{array}{c}
\rho_{j}^{0} \\
\rho_{j}^{0} V \\
\rho_{j}^{0} g_{j}^{0}
\end{array}\right)+\left(\begin{array}{c}
0 \\
P \\
0
\end{array}\right) .
$$

Expression (18) is also valid for any approximate Riemann solver that features an exact representation of contact discontinuities, such as Osher's scheme. From Eqs. (17)-(18) it follows that

$$
\mathbf{q}_{j}^{1}=\mathbf{q}_{j}^{0}-C\left(\mathbf{q}_{j}^{0}-\mathbf{q}_{j-1}^{0}\right)
$$

with

$$
C:=V\left(t_{1}-t_{0}\right) /\left(x_{j+1}-x_{j}\right),
$$

the local CFL-number. From Eqs. (19) and (13b) we obtain, successively,

$$
\rho_{j}^{1}=\rho_{j}^{0}-C\left(\rho_{j}^{0}-\rho_{j-1}^{0}\right)=g_{j}^{*} \rho_{\mathrm{p}}(P)+\left(1-g_{j}^{*}\right) \rho_{\mathrm{s}}(P),
$$

with

$$
g_{j}^{*}:=g_{j}^{0}-C\left(g_{j}^{0}-g_{j-1}^{0}\right) .
$$

Comparing (20) to (13b), we infer that a necessary and sufficient condition for pressure invariance of the discrete approximation is $g_{j}^{1}=g_{j}^{*}$. However, conversely, from (13b) and (19) we obtain

$$
g_{j}^{1}=\frac{\beta_{2} \rho_{\mathrm{p}}+\left(\beta_{1}-\beta_{2}\right) \rho_{\mathrm{s}}}{\beta_{1} \rho_{\mathrm{p}}+\left(1-\beta_{1}\right) \rho_{\mathrm{s}}},
$$

with

$$
\begin{gathered}
\beta_{1}=(1-C) g_{j}^{0}+C g_{j-1}^{0}, \\
\beta_{2}=(1-C)\left(g_{j}^{0}\right)^{2}+C\left(g_{j-1}^{0}\right)^{2},
\end{gathered}
$$

with $\rho_{\mathrm{p} / \mathrm{s}}:=\rho_{\mathrm{p} / \mathrm{s}}(P)$. In general, $g_{j}^{1} \neq g_{j}^{*}$ and, hence, the discrete approximation from (17) lacks the pressureinvariance property of the continuum equations (9). Trivial exceptions are: $C=0\left(\Rightarrow \mathbf{q}_{j}^{1}=\mathbf{q}_{j}^{0}\right), C=1\left(\Rightarrow \mathbf{q}_{j}^{1}=\right.$ $\left.\mathbf{q}_{j-1}^{0}\right), g_{j}^{0}=g_{j-1}^{0}\left(\Rightarrow \mathbf{q}_{j}^{0}=\mathbf{q}_{j-1}^{0}\right)$ and $\rho_{\mathrm{p}}=\rho_{\mathrm{s}}$.
It is noteworthy that if $(\rho g)_{t}+(\rho g v)_{x}=0$ in (9) is replaced by

$$
g_{t}+v g_{x}=0, \quad x \in \mathcal{L}, t \geq 0,
$$

then, subject to the initial conditions (13), the first-order forward Euler discretization yields

$$
g_{j}^{1}=g_{j}^{0}-C\left(g_{j}^{0}-g_{j-1}^{0}\right) .
$$

Hence, $g_{j}^{1}=g_{j}^{*}$, and pressure invariance is maintained. However, Eq. (22) is in non-conservative form. The pressure invariance is in this case achieved at the expense of the conservative form of the equations.

\section{Pressure-Invariance Condition}

The implications of the above exemplification are restricted: The analysis does not imply that pressure oscillations are inherent to conservative discretizations of twofluid flow problems. It merely implies that discrete approximations to two-fluid flow problems do not necessarily inherit the pressure-invariance property of the continuum equations.

To avoid pressure oscillations, discrete approximations of two-fluid flow problems must comply with a pressureinvariance condition. This condition is also mentioned in Ref. ${ }^{24}$ in the context of a not-strictly-conservative method for multi-fluid flows with a stiffened-gas equation of state; see also ${ }^{3,25,26}$. Below we formulate the pressureinvariance condition for strictly conservative hyperbolic systems conform (9), provided with a compound equation of state of the form $p(\rho, \theta)$. We do not yet attach a specific connotation to $g$.

The pressure-invariance condition for discretizations of (9) is stated: If $v_{j}^{k}=V$, with $V$ a constant, and $\rho_{j}^{k}$ and $\theta_{j}^{k}$ satisfy

$$
p\left(\rho_{j}^{k}, \theta_{j}^{k}\right)=P,
$$

for some constant $P$, then $p$ is invariant under the characteristic mapping of the discretization, i.e.,

$$
p\left(\rho_{j}^{k+1}, \theta_{j}^{k+1}\right)=P .
$$

In fact, $g_{j}^{1}=g_{j}^{*}$ with $g_{j}^{*}$ according to Eq. (20b) is an implementation of the pressure-invariance condition for a compound equation of state conform (12) and the firstorder forward Euler discretization (17).

\section{A Non-Oscillatory Conservative Scheme}

To set up a pressure-invariant discretization for two-fluid flow problems, we consider two distinct compressible fluids with barotropic equations of state $\rho_{\mathrm{p}}(p)$ and $\rho_{\mathrm{s}}(p)$. For given density and pressure, the primary volume fraction $\alpha$ is implicitly defined by

$$
\rho(x, t)=\alpha(x, t) \rho_{\mathrm{p}}(p(x, t))+(1-\alpha(x, t)) \rho_{\mathrm{s}}(p(x, t)) .
$$

Under the assumption $\rho_{\mathrm{p}}(p) \neq \rho_{\mathrm{s}}(p)$, Eq. (25) uniquely defines $\alpha$. However, $\alpha$ does not appear in our final formulation and we do not rely on its unicity. 
We also require the primary and secondary partial densities, defined as:

$$
\rho_{\mathrm{p}}^{\prime}:=\alpha \rho_{\mathrm{p}}, \quad \text { and } \quad \rho_{\mathrm{s}}^{\prime}:=(1-\alpha) \rho_{\mathrm{s}},
$$

respectively. In terms of these partial densities, conservation of mass, for each fluid separately, is expressed by

$$
\left(\rho_{\mathrm{p}}^{\prime}\right)_{t}+\left(\rho_{\mathrm{p}}^{\prime} v\right)_{x}=0, \quad \text { and } \quad\left(\rho_{\mathrm{s}}^{\prime}\right)_{t}+\left(\rho_{\mathrm{s}}^{\prime} v\right)_{x}=0 .
$$

Furthermore, the compound density satisfies $\rho=\rho_{\mathrm{p}}^{\prime}+\rho_{\mathrm{s}}^{\prime}$. Hence, if we assign $g$ as the primary mass fraction,

$$
g:=\rho_{\mathrm{p}}^{\prime} / \rho
$$

then conservation of mass, for each of the fluids separately, and conservation of momentum can be condensed into the form (9).

The compound equation of state associated with $g$ according to (28) is implicitly given by

$$
\begin{aligned}
\rho g & =\alpha \rho_{\mathrm{p}}(p), \\
\rho-\rho g & =(1-\alpha) \rho_{\mathrm{s}}(p) .
\end{aligned}
$$

Eq. (29) follows from $\rho g=\rho_{\mathrm{p}}^{\prime}$ and $\rho-\rho g=\rho_{\mathrm{S}}^{\prime}$ and (26). Elimination of $\alpha$ yields the convenient form

$$
\frac{1}{\rho}=\frac{g}{\rho_{\mathrm{p}}(p)}+\frac{1-g}{\rho_{\mathrm{s}}(p)} .
$$

The first-order forward Euler discretization of (9) with the compound equation of state (29) or (30) satisfies the pressure-invariance condition. To corroborate this assertion, we note that if $v_{j}^{k}=V$ and $p\left(\rho_{j}^{k}, g_{j}^{k}\right)=P$, i.e.,

$$
\begin{aligned}
\rho_{j}^{k} g_{j}^{k} & =\alpha_{j}^{k} \rho_{\mathrm{p}}(P), \\
\rho_{j}^{k}-\rho_{j}^{k} g_{j}^{k} & =\left(1-\alpha_{j}^{k}\right) \rho_{\mathrm{s}}(P),
\end{aligned}
$$

for all $j=1, \ldots, n$, then the forward Euler discretization (17) with the numerical flux (18) yields

$$
\begin{aligned}
\rho_{j}^{k+1} & =\rho_{j}^{k}-C\left(\rho_{j}^{k}-\rho_{j-1}^{k}\right), \\
\rho_{j}^{k+1} g_{j}^{k+1} & =\rho_{j}^{k} g_{j}^{k}-C\left(\rho_{j}^{k} g_{j}^{k}-\rho_{j-1}^{k} g_{j-1}^{k}\right),
\end{aligned}
$$

with $C$ defined by (19b). From (31)-(32) it follows that

$$
\begin{aligned}
\rho_{j}^{k+1} g_{j}^{k+1} & =\alpha_{j}^{k+1} \rho_{\mathrm{p}}(P), \\
\rho_{j}^{k+1}-\rho_{j}^{k+1} g_{j}^{k+1} & =\left(1-\alpha_{j}^{k+1}\right) \rho_{\mathrm{s}}(P),
\end{aligned}
$$

with

$$
\alpha_{j}^{k+1}:=\alpha_{j}^{k}-C\left(\alpha_{j}^{k}-\alpha_{j-1}^{k}\right) .
$$

The compound equation of state yields $p\left(\rho_{j}^{k+1}, g_{j}^{k+1}\right)=P$.

thus

Summarizing, we conclude that if $g$ represents the primary volume fraction and the compound equation of state is specified accordingly as (12), then the discretization does not comply with the pressure-invariance condition. In contrast, if $g$ is the primary mass fraction and the compound equation of state is given by (30), then the pressureinvariance condition is satisfied.

\section{A Modified, Two-Fluid OSher SCHEME}

By virtue of its conservative form, the above pressureinvariant formulation, based on the mass fraction, is ideally suited to treatment by Godunov-type methods. To avoid the computational expenses of solving the associated Riemann problems, below we set up an approximate Riemann solver for the two-fluid flow problem. The approximate Riemann solver is of Osher type. As a digression, we show that the interface indeed appears as a contact discontinuity, both in the exact Riemann solution and in the rarefaction-waves-only approximation that underlies Osher's scheme.

We emphasize that the choice of the approximate Riemann solver does not affect the pressure invariance; the invariance is ensured by the specific choice (28) for $g$ and the corresponding compound equation of state (30). Any other approximate Riemann solver that resolves contact discontinuities exactly could have been selected here, e.g., Roe's scheme or the AUSM scheme.

\section{The Two-Fluid Riemann Problem}

We consider (9) provided with a compound equation of state of the form $p:=p(\rho, g)$, e.g., Eq. (30). The formal dependence of $g$ on $\theta$ in (9) can be ignored. The corresponding Riemann problem is defined on the half-space $\Omega:=\{-\infty<x<\infty, 0<t<\infty\}$ and is obtained by imposing the discontinuous initial conditions

$$
\mathbf{q}(x, 0):= \begin{cases}\mathbf{q}_{L} & \text { if } x<0 \\ \mathbf{q}_{R} & \text { otherwise }\end{cases}
$$

for certain constant left and right states $\mathbf{q}_{L}$ and $\mathbf{q}_{R}$.

The properties of the Riemann problem and its solution are classical; see, e.g., ${ }^{27}$. This paragraph serves to collect the essentials for the ensuing presentation and contains the specifics for the two-fluid flow problem.

To obtain the Riemann solution for the two-fluid Euler equations, we need the Jacobian $\mathbf{A}(\mathbf{q}$ of $\mathbf{f}(\mathbf{q})$ :

$$
\mathbf{A}(\mathbf{q}):=\frac{\partial \mathbf{f}(\mathbf{q})}{\partial \mathbf{q}}=\left(\begin{array}{ccc}
0 & 1 & 0 \\
-\left(q_{2}^{2}+c_{2}^{2} q_{3}\right) / q_{1}^{2}+c_{1}^{2} & 2 q_{2} / q_{1} & c_{2}^{2} / q_{1} \\
-q_{3} q_{2} / q_{1}^{2} & q_{3} / q_{1} & q_{2} / q_{1}
\end{array}\right)
$$

with

$$
c_{1}(\rho, g):=\sqrt{\partial p(\rho, g) / \partial \rho}, \quad c_{2}(\rho, g):=\sqrt{\partial p(\rho, g) / \partial g} .
$$

Its eigenvalues are

$$
\lambda_{1}:=q_{2} / q_{1}-c_{1}, \quad \lambda_{2}:=q_{2} / q_{1}, \quad \lambda_{3}:=q_{2} / q_{1}+c_{1},
$$


and the corresponding eigenvectors are

$$
\begin{aligned}
\mathbf{r}_{1}:=\left(\begin{array}{c}
1 \\
q_{2} / q_{1}-c_{1} \\
q_{3} / q_{1}
\end{array}\right), \quad \mathbf{r}_{2}:=\left(\begin{array}{c}
q_{1} \\
q_{2} \\
-\left(c_{1} / c_{2}\right)^{2} q_{1}^{2}+q_{3}
\end{array}\right) \\
\mathbf{r}_{3}:=\left(\begin{array}{c}
1 \\
q_{2} / q_{1}+c_{1} \\
q_{3} / q_{1}
\end{array}\right)
\end{aligned}
$$

The eigenpairs $\left(\lambda_{k}, \mathbf{r}_{k}\right)$ are genuinely nonlinear for $k=$ 1,3 and linearly degenerate for $k=2$ (cf. Ref. ${ }^{18}$ for a definition of these classifications). The genuinely nonlinear eigenpairs are related to rarefaction waves and shock waves. The linearly degenerate eigenpair corresponds to a contact discontinuity.

For any admissible state $\mathbf{q}_{A}$ we associate two paths in state space with each eigenpair: the $k$-shock path and the $k$-rarefaction path. The $k$-shock path is defined as

$$
\begin{array}{r}
\mathcal{S}_{k}\left(\mathbf{q}_{A}\right):=\left\{\mathbf{q} \in \mathbb{R}^{3}: s\left(\mathbf{q}, \mathbf{q}_{A}\right)\left(\mathbf{q}-\mathbf{q}_{A}\right)=\mathbf{f}(\mathbf{q})-\mathbf{f}\left(\mathbf{q}_{A}\right),\right. \\
\left.s\left(\mathbf{q}, \mathbf{q}_{A}\right) \rightarrow \lambda_{k}\left(\mathbf{q}_{A}\right) \text { as } \mathbf{q} \rightarrow \mathbf{q}_{A}\right\},
\end{array}
$$

where $s\left(\mathbf{q}, \mathbf{q}_{A}\right)$ is referred to as the $k$-shock speed. The $k$-rarefaction path is defined as

$$
\mathcal{R}_{k}\left(\mathbf{q}_{A}\right):=\left\{\mathbf{q} \in \mathbb{R}^{3}: \mathbf{q}=\mathbf{h}(\xi), \xi \in \mathbb{R}\right\},
$$

with $\mathbf{h}(\xi)$ the solution to the ordinary differential equation $\mathbf{h}^{\prime}(\xi)=\mathbf{r}_{k}(\mathbf{h}(\xi)) / \beta(\mathbf{h}(\xi))$, subject to $\mathbf{h}\left(\lambda_{k}\left(\mathbf{q}_{A}\right)\right)=\mathbf{q}_{A}$,

with $\beta:=\partial_{\mathbf{q}} \lambda_{k}(\mathbf{q}) \cdot \mathbf{r}_{k}(\mathbf{q})$ for the genuinely nonlinear eigenpairs and $\beta:=1$ for the linearly degenerate eigenpair. Note that $\lambda_{k}(\mathbf{h}(\xi))=\xi$ for the genuinely nonlinear eigenpairs.

The Riemann solution can be constructed by means of the shock and rarefaction paths. The solution is constant in four (possibly empty) disjoint subsets of $\Omega$. The constant states are denoted by $\mathbf{q}_{k / 3}, k=0,1,2,3$. Furthermore, we set $\mathbf{q}_{0}:=\mathbf{q}_{L}$ and $\mathbf{q}_{1}:=\mathbf{q}_{R}$. We refer to $\mathbf{q}_{1 / 3}$ and $\mathbf{q}_{2 / 3}$ as intermediate states. By connecting each pair of consecutive states by either a shock or a rarefaction path, we can connect $\mathbf{q}_{0}$ to $\mathbf{q}_{1}$. The unique sequence of paths that satisfies $\lambda_{k}\left(\mathbf{q}_{(k-1) / 3}\right)>\lambda_{k}\left(\mathbf{q}_{k / 3}\right)$ if $\mathbf{q}_{(k-1) / 3}$ and $\mathbf{q}_{k / 3}$ are connected by $\mathcal{S}_{k}$ and $\lambda_{k}\left(\mathbf{q}_{(k-1) / 3}\right) \leq \lambda_{k}\left(\mathbf{q}_{k / 3}\right)$ if $\mathbf{q}_{(k-1) / 3}$ and $\mathbf{q}_{k / 3}$ are connected by $\mathcal{R}_{k}$ corresponds to the Riemann solution. If $\lambda_{k}\left(\mathbf{q}_{(k-1) / 3}\right)=\lambda_{k}\left(\mathbf{q}_{k / 3}\right)$ then the shock and rarefaction paths coincide and we opt for a rarefaction-path connection. This situation occurs for the contact discontinuity.

Recalling that the Riemann solution assumes the similarity form $\mathbf{q}(x, t)=\mathbf{q}(x / t)$ (see, e.g., Ref. ${ }^{27}$ ), we obtain

$$
\mathbf{q}(x, t):=\mathbf{q}(x / t)= \begin{cases}\mathbf{q}_{0} & \text { if } x / t<\sigma_{0}^{+}, \\ \mathbf{q}_{k / 3} & \text { if } \sigma_{k}^{-}<x / t<\sigma_{k}^{+}, \\ \mathbf{h}_{k}(x / t) & \text { if } \sigma_{k-1}^{+}<x / t<\sigma_{k}^{-}, \\ \mathbf{q}_{1} & \text { if } x / t>\sigma_{3}^{-},\end{cases}
$$

where $\mathbf{h}_{k}:=\mathbf{h}$ according to (39b) with $\mathbf{q}_{A}:=\mathbf{q}_{(k-1) / 3}$ and

$\sigma_{k}^{+}= \begin{cases}\lambda_{k+1}\left(\mathbf{q}_{k / 3}\right), & \text { if } \lambda_{k+1}\left(\mathbf{q}_{k / 3}\right) \leq \lambda_{k+1}\left(\mathbf{q}_{(k+1) / 3}\right), \\ s_{k+1}, & \text { otherwise }\end{cases}$

$\sigma_{k}^{-}= \begin{cases}\lambda_{k}\left(\mathbf{q}_{k / 3}\right), & \text { if } \lambda_{k}\left(\mathbf{q}_{k / 3}\right) \geq \lambda_{k}\left(\mathbf{q}_{(k-1) / 3}\right), \\ s_{k}, & \text { otherwise. }\end{cases}$

An example of the solution (40) is presented in Figure 2.

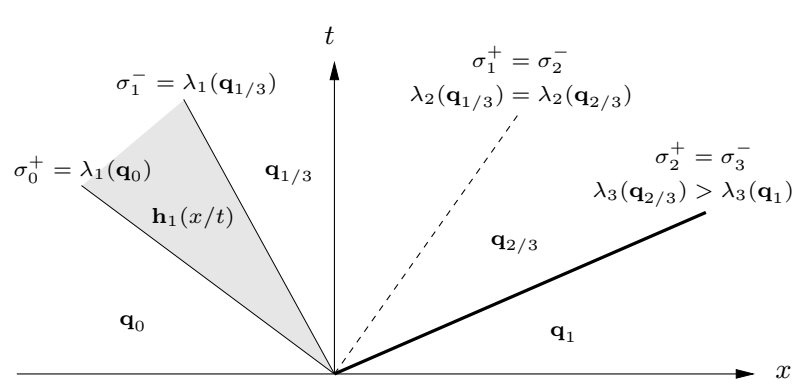

Figure 2: Illustration of a two-fluid Riemann solution: An expansion fan (shaded) connects $\mathbf{q}_{0}$ to $\mathbf{q}_{1 / 3}$, a contact discontinuity (dashed) connects $\mathbf{q}_{1 / 3}$ to $\mathbf{q}_{2 / 3}$ and a shock discontinuity (solid) connects $\mathbf{q}_{2 / 3}$ to $\mathbf{q}_{1}$.

\section{RIEMANN INVARIANTS}

To each $k$-rarefaction path corresponds a set of Riemann invariants, i.e., functions which are invariant on $\mathcal{R}_{k}$. These Riemann invariants allow us to conveniently determine the intermediate states in the rarefaction-waves-only approximation to the Riemann solution that underlies Osher's scheme. Moreover, by means of the Riemann invariants and a simple argument for shocks, we can show that the interface indeed appears as a contact discontinuity.

Consider the eigenvectors (37). A $k$-Riemann invariant for the two-fluid Euler equations (9) is any continuously differentiable function $\psi_{k}: \mathbb{R}^{3} \mapsto \mathbb{R}$ with the property

$$
\partial_{\mathbf{q}} \psi_{k}(\mathbf{q}) \cdot \mathbf{r}_{k}(\mathbf{q})=0 .
$$

There are at most two such $k$-Riemann invariants with linearly independent partial derivatives. Note that for the linearly degenerate eigenpair the eigenvalue is a Riemann invariant.

To derive the 1-Riemann invariants, we first solve the system of ordinary differential equations

$$
\mathbf{h}^{\prime}(\xi)=\mathbf{r}_{k}(\mathbf{h}(\xi)), \quad \text { subject to } \quad \mathbf{h}(0)=\mathbf{h}^{0},
$$

with $k=1$ :

$$
\begin{aligned}
& h_{1}(\xi)=\xi+h_{1}^{0}, \\
& h_{2}(\xi)=h_{1}(\xi)\left(\frac{h_{2}^{0}}{h_{1}^{0}}-\int_{h_{1}^{0}}^{h_{1}(\xi)} \frac{c_{1}(\omega)}{\omega} d \omega\right), \\
& h_{3}(\xi)=\left(h_{3}^{0} / h_{1}^{0}\right) \xi+h_{3}^{0},
\end{aligned}
$$


with $c_{1}(\omega):=c_{1}\left(h_{1}(\omega), h_{3}(\omega) / h_{1}(\omega)\right)$. The 1-Riemann invariants can be obtained by constructing $\xi$-independent functions of $h_{j}(\xi), j=1,2,3$. The invariants thus obtained are presented in (48). Note that by virtue of the similitude of $\mathbf{r}_{1}$ and $\mathbf{r}_{3}$, the 3-Riemann invariants can be chosen identical to the 1-Riemann invariants with $c_{1}$ replaced by $-c_{1}$.

To derive the 2-Riemann invariants, we solve (42) for $k=2$. Obviously,

$$
h_{1}(\xi)=h_{1}^{0} \mathrm{e}^{\xi}, \quad \text { and } \quad h_{2}(\xi)=h_{2}^{0} \mathrm{e}^{\xi} .
$$

To determine $h_{3}(\xi)$, we recall that $c_{1}$ and $c_{2}$ are defined by (35b). Therefore, Eq. (42) yields

$$
h_{3}^{\prime} D_{2} p+D_{1} p-h_{3} D_{2} p=0
$$

where $D_{j}$ denotes differentiation with respect to the $j$-th argument. Moreover, from $p:=p\left(h_{1}, h_{3} / h_{1}\right)$ we obtain

$$
\frac{d p}{d \xi}=h_{1}^{\prime}\left(D_{1} p-\frac{h_{3} D_{2} p}{h_{1}^{2}}\right)+h_{3}^{\prime} \frac{D_{2} p}{h_{1}} .
$$

Eqs. (44)-(46) imply that $d p / d \xi=0$, i.e., $p$ is a 2 Riemann invariant and $h_{3}(\xi)$ is implicitly specified by

$$
p\left(h_{1}(\xi), h_{3}(\xi) / h_{1}(\xi)\right)=p\left(h_{1}^{0}, h_{3}^{0} / h_{1}^{0}\right) .
$$

From (44)-(47) we infer that $p$ and $q_{2} / q_{1}$ are 2-Riemann invariants. Indeed, the linearly degenerate eigenvalue $\lambda_{2}:=q_{2} / q_{1}$ is a 2 -Riemann invariant.

Summarizing, we can associate the following Riemann invariants with the two-fluid Euler equations (9) with a compound equation of state of the form $p:=p(\rho, g)$ :

$$
\begin{array}{lll}
\psi_{1}^{2}=v+\Psi(\rho, g), & \psi_{2}^{1}=v, & \psi_{3}^{1}=v-\Psi(\rho, g) \\
\psi_{1}^{3}=g, & \psi_{2}^{3}=p, & \psi_{3}^{2}=g
\end{array}
$$

where

$$
\Psi(\rho, g):=\int_{\rho^{0}}^{\rho} \frac{c_{1}(\omega, g)}{\omega} d \omega,
$$

with $\rho^{0}$ an arbitrary positive real constant.

It is important to note that $g$ is a Riemann invariant for the genuinely nonlinear eigenpairs $(k=1,3)$ and that $p$ and $v$ are Riemann invariants for the linearly degenerate eigenpair $(k=2)$. In the absence of shocks, this implies that the change in $g$ associated with the fluid transition at the interface can only occur across the contact discontinuity and, moreover, that the interface conditions (4) are indeed satisfied.

To demonstrate that $g$ is also invariant across genuine (non-degenerate) shocks, we note that

$$
\begin{aligned}
s\left(\rho-\rho_{A}\right) & =\rho v-\rho_{A} v_{A} \Rightarrow \\
s\left(\rho g_{A}-\rho_{A} g_{A}\right) & =\rho g_{A} v-\rho_{A} g_{A} v_{A},
\end{aligned}
$$

for any constant $g_{A}$. From (38) and (49) we can infer that there exist two shock paths on which $g$ is invariant. Moreover, the shock path and rarefaction path of the degenerate shock $(k=2)$ coincide. Because $g$ is not a 2-Riemann invariant, $g$ can vary on the 2 -shock path. Therefore, the shock paths on which $g$ is invariant must be the 1-and 3 -shock paths. These paths correspond to genuine shocks. The invariance of $g$ on the 1- and 3-shock paths implies that the fluid transition at the interface cannot occur across a genuine shock.

\section{RAREFACTION-WAVES-ONLY APPROXIMATION}

In the foregoing it was shown that the intermediate states in the Riemann solution are connected by shock and rarefaction paths. A rarefaction-waves-only approximation is obtained by replacing the shock paths by rarefaction paths. Shock discontinuities in the Riemann solution are then approximated by so-called overturned rarefaction waves; see, e.g., Ref. ${ }^{19}$.

The intermediate states in the rarefaction-waves-only approximation can be conveniently determined by means of the Riemann invariants. Supposing the approximate intermediate states $\tilde{\mathbf{q}}_{(l-1) / n}$ and $\tilde{\mathbf{q}}_{l / n}$ are connected by $\mathcal{R}_{k(l)}$, with $k:\{1,2,3\} \mapsto\{1,2,3\}$ a bijection,

$$
\begin{aligned}
\psi_{k(l)}^{m}\left(\tilde{\mathbf{q}}_{(l-1) / 3}\right)= & \psi_{k(l)}^{m}\left(\tilde{\mathbf{q}}_{l / 3}\right), l, m=1,2,3, m \neq k(l), \\
& \text { with } \tilde{\mathbf{q}}_{0}:=\mathbf{q}_{L} \text { and } \tilde{\mathbf{q}}_{1}:=\mathbf{q}_{R} .
\end{aligned}
$$

Usual choices for the ordering of the paths are the O-variant $k(l):=4-l$ (see Ref. ${ }^{22}$ ) and the P-variant $k(l):=l$ (see Ref. ${ }^{10}$ ). The O-variant and the P-variant have mutually reversed orderings. Throughout, we presume a P-variant ordering.

Eq. (50) represents a system of nonlinear equations, from which the approximate intermediate states $\tilde{\mathbf{q}}_{1 / 3}$ and $\tilde{\mathbf{q}}_{2 / 3}$ have to be extracted. Using the expressions for the Riemann invariants (48), it is easy to show that the Jacobian matrix corresponding to (50) is nonsingular. Therefore, by the inverse function theorem, Eq. (50) is indeed solvable.

To establish the accuracy of the approximate intermediate states from (50), we recall from ${ }^{27}$ that the change in the $k$-Riemann invariants across a $k$-shock with strength $\mu$ is $O\left(\mu^{3}\right)$ as $\mu \rightarrow 0$, with the $k$-shock strength defined as the change in the eigenvalue $\lambda_{k}$ across the shock. It follows that for sufficiently weak shocks, i.e., if $\mu:=$ $\sup _{k=1,3}\left(\lambda_{k}\left(\mathbf{q}_{(k-1) / 3}\right)-\lambda_{k}\left(\mathbf{q}_{k / 3}\right)\right)$ is sufficiently small, the error in the approximate intermediate states is only $O\left(\mu^{3}\right)$ as well. Moreover, in the absence of shocks, the approximation according to (50) is even exact. If strong shocks impair the accuracy of the numerical solution, then an approximate Riemann solver which is suitable for shocks, or even an exact Riemann solver, should be applied.

From (48) and (50) we obtain

$$
\tilde{g}_{1 / 3}=g_{L}, \quad \tilde{g}_{2 / 3}=g_{R}, \text { and } \tilde{v}_{1 / 3}=\tilde{v}_{2 / 3}=: \tilde{v}_{1 / 2},
$$

and, in turn,

$$
\tilde{v}_{1 / 2}+\int_{\rho_{L}}^{\tilde{\rho}_{1 / 3}} \frac{c_{1}\left(\rho, g_{L}\right)}{\rho} d \rho=v_{L},
$$




$$
\begin{array}{r}
\tilde{v}_{1 / 2}-\int_{\rho_{R}}^{\tilde{\rho}_{2 / 3}} \frac{c_{1}\left(\rho, g_{R}\right)}{\rho} d \rho=v_{R}, \\
p\left(\tilde{\rho}_{1 / 3}, g_{L}\right)=p\left(\tilde{\rho}_{2 / 3}, g_{R}\right), \quad\left(=: \tilde{p}_{1 / 2}\right) .
\end{array}
$$

For a compound equation of state of the form $\rho:=\rho(p, g)$, e.g., Eq. (30), these conditions for the intermediate states can be cast in a convenient form. To derive this form, we use Eq. (35b) and the transformation $\rho:=\rho(p, \theta)$ to obtain, successively,

$$
\begin{aligned}
\int_{\rho_{a}}^{\rho_{b}} \frac{c_{1}(\rho, g)}{\rho} d \rho= & \int_{\rho_{a}}^{\rho_{b}} \frac{1}{\rho} \sqrt{\frac{\partial p(\rho, g)}{\partial \rho}} d \rho= \\
& \int_{p_{a}}^{p_{b}} \frac{1}{\rho(p, g)} \sqrt{\frac{\partial \rho(p, g)}{\partial p}} d p
\end{aligned}
$$

for any $\rho_{a}, \rho_{b} \in \mathbb{R}_{+}$and corresponding $p_{a}, p_{b}$. Eqs. (52)(53) imply

$$
\begin{aligned}
& \int_{p_{L}}^{\tilde{p}_{1 / 2}} \frac{1}{\rho\left(p, g_{L}\right)} \sqrt{\frac{\partial \rho\left(p, g_{L}\right)}{\partial p}} d p+ \\
& \quad \int_{p_{R}}^{\tilde{p}_{1 / 2}} \frac{1}{\rho\left(p, g_{R}\right)} \sqrt{\frac{\partial \rho\left(p, g_{R}\right)}{\partial p}} d p=v_{L}-v_{R} .
\end{aligned}
$$

Equation (54) presents a concise condition for the intermediate pressure $\tilde{p}_{1 / 2}$. Once the intermediate pressure has been extracted from (54), the intermediate densities follow from the compound equation of state and $\tilde{v}_{1 / 2}$ is obtained from (52a) or (52b) in a straightforward manner.

It is noteworthy that (54) is well suited to treatment by numerical approximation techniques. In particular, the derivatives of the integrals with respect to $\tilde{p}_{1 / 2}$, which are required in Newton's method, are simply the integrands evaluated at $\tilde{p}_{1 / 2}$. Moreover, for a given approximation to $\tilde{p}_{1 / 2}$, the integrals can be evaluated by a standard numerical integration method (see, e.g., Ref. ${ }^{12}$ ).

\section{THE MOdIFIED OSHER SCHEME}

The numerical flux in Osher's scheme ${ }^{22}$, is determined by

$$
\mathbf{f}_{O}\left(\mathbf{q}_{L}, \mathbf{q}_{R}\right):=\frac{1}{2} \mathbf{f}\left(\mathbf{q}_{L}\right)+\frac{1}{2} \mathbf{f}\left(\mathbf{q}_{R}\right)-\frac{1}{2} \sum_{l=1}^{3} \mathbf{d}_{l},
$$

with

$$
\mathbf{d}_{l}:=\int_{0}^{1}|\mathbf{A}(\mathbf{h}(\xi))| \cdot \mathbf{r}_{k(l)}(\mathbf{h}(\xi)) d \xi
$$

where $\mathbf{h}(\xi)$ refers to a parametrization of the section of the $k(l)$-rarefaction path between $\tilde{\mathbf{q}}_{(l-1) / 3}$ and $\tilde{\mathbf{q}}_{l / 3}$ and

$$
|\mathbf{A}(\mathbf{q})|:=\left(\mathbf{r}_{1}, \mathbf{r}_{2}, \mathbf{r}_{3}\right) \cdot \operatorname{diag}\left(\left|\lambda_{1}\right|,\left|\lambda_{2}\right|,\left|\lambda_{3}\right|\right) \cdot\left(\mathbf{r}_{1}, \mathbf{r}_{2}, \mathbf{r}_{3}\right)^{-1},
$$

with the eigenvalues and eigenvectors according to (36) and (37), their dependence on $\mathbf{q}$ being suppressed for transparency. The numerical flux (55) approximates $\mathbf{f}(\mathbf{q}(0))$, with $\mathbf{q}(x / t)$ the Riemann solution in similarity form according to (40).
From Eqs. (55b)-(55c) it follows that

$$
\mathbf{d}_{l}=\int_{0}^{1} \operatorname{sign}\left(\lambda_{k(l)}(\mathbf{h}(\xi))\right) \mathbf{A}(\mathbf{h}(\xi)) \cdot \mathbf{r}_{k(l)}(\mathbf{h}(\xi)) d \xi .
$$

If $\lambda_{k(l)}$ in (56) does not change sign on the integration interval, then the integral evaluates to

$$
\mathbf{d}_{l}=\operatorname{sign}\left(\lambda_{k(l)}\left(\tilde{\mathbf{q}}_{(l-1) / n}\right)\right)\left(\mathbf{f}\left(\tilde{\mathbf{q}}_{l / n}\right)-\mathbf{f}\left(\tilde{\mathbf{q}}_{(l-1) / n}\right)\right),
$$

whereas if $\lambda_{k(l)}$ changes its sign once, say at $\tilde{\mathbf{q}}_{*}$ (i.e., $\left.\lambda_{k(l)}\left(\tilde{\mathbf{q}}_{*}\right)=0\right)$, then

$$
\begin{aligned}
& \mathbf{d}_{l}=\operatorname{sign}\left(\lambda_{k(l)}\left(\tilde{\mathbf{q}}_{(l-1) / n}\right)\right) \\
& \left(\left(\mathbf{f}\left(\tilde{\mathbf{q}}_{*}\right)-\mathbf{f}\left(\tilde{\mathbf{q}}_{(l-1) / n}\right)\right)-\left(\mathbf{f}\left(\tilde{\mathbf{q}}_{l / n}\right)-\mathbf{f}\left(\tilde{\mathbf{q}}_{*}\right)\right)\right) .
\end{aligned}
$$

Under the condition $0<\lambda_{2}\left(\tilde{\mathbf{q}}_{1 / 3}\right)=\lambda_{2}\left(\tilde{\mathbf{q}}_{2 / 3}\right)<$ $\lambda_{3}\left(\tilde{\mathbf{q}}_{2 / 3}\right), \lambda_{3}\left(\tilde{\mathbf{q}}_{1}\right)$, we can then derive three generic cases

$$
\begin{gathered}
\mathbf{f}_{O}\left(\mathbf{q}_{L}, \mathbf{q}_{R}\right)= \\
\begin{cases}\mathbf{f}\left(\tilde{\mathbf{q}}_{*}\right) & \text { if } \lambda_{1}\left(\tilde{\mathbf{q}}_{0}\right)<0<\lambda_{1}\left(\tilde{\mathbf{q}}_{1 / 3}\right), \\
\mathbf{f}\left(\tilde{\mathbf{q}}_{1 / 3}\right) & \text { if } \lambda_{1}\left(\tilde{\mathbf{q}}_{0}\right)<\lambda_{1}\left(\tilde{\mathbf{q}}_{1 / 3}\right)<0, \\
\mathbf{f}\left(\tilde{\mathbf{q}}_{0}\right)+\mathbf{f}\left(\tilde{\mathbf{q}}_{1 / 3}\right)-\mathbf{f}\left(\tilde{\mathbf{q}}_{*}\right) & \text { if } \lambda_{1}\left(\tilde{\mathbf{q}}_{0}\right)>0>\lambda_{1}\left(\tilde{\mathbf{q}}_{1 / 3}\right) .\end{cases}
\end{gathered}
$$

Comparison to the corresponding $\mathbf{f}(\mathbf{q}(0))$ shows that $\mathbf{f}_{O}\left(\mathbf{q}_{L}, \mathbf{q}_{R}\right)$ is accurate in the first two cases, in particular, the error is then $O\left(\mu^{3}\right)$, and inaccurate in the third case, the error then being $O(\mu)$; see also ${ }^{4}$. This failure of Osher's scheme is exemplified by means of the Burgers equation in ${ }^{19}$.

To avoid the aforementioned deficiency of Osher's scheme, we propose a modification of the scheme. The rarefaction-waves-only approximation is maintained. However, the overturned-rarefaction-wave representation of shocks in the approximate Riemann solution is avoided. Instead, the intermediate states from (50), with a presumed $\mathrm{P}$-variant ordering of the subpaths, are used to construct the approximate Riemann solution:

$$
\tilde{\mathbf{q}}(x / t):= \begin{cases}\tilde{\mathbf{q}}_{0} & \text { if } x / t<\tilde{\sigma}_{0}^{+}, \\ \tilde{\mathbf{q}}_{k / 3} & \text { if } \tilde{\sigma}_{k}^{-}<x / t<\tilde{\sigma}_{l}^{+}, \\ \mathbf{h}_{k}(x / t) & \text { if } \tilde{\sigma}_{k-1}^{+}<x / t<\tilde{\sigma}_{k}^{-}, \\ \tilde{\mathbf{q}}_{1} & \text { if } x / t>\tilde{\sigma}_{3}^{-},\end{cases}
$$

where $\mathbf{h}_{k}:=\mathbf{h}$ according to (39b) with $\mathbf{q}_{A}:=\tilde{\mathbf{q}}_{(k-1) / 3}$ and

$\tilde{\sigma}_{k}^{+}:= \begin{cases}\lambda_{k+1}\left(\tilde{\mathbf{q}}_{k / 3}\right) & \text { if } \lambda_{k+1}\left(\tilde{\mathbf{q}}_{k / 3}\right) \leq \lambda_{k+1}\left(\tilde{\mathbf{q}}_{(k+1) / 3}\right), \\ \tilde{s}_{k+1} & \text { otherwise }\end{cases}$

$$
\tilde{\sigma}_{k}^{-}:= \begin{cases}\lambda_{k}\left(\tilde{\mathbf{q}}_{k / 3}\right) & \text { if } \lambda_{k}\left(\tilde{\mathbf{q}}_{k / 3}\right) \geq \lambda_{k}\left(\tilde{\mathbf{q}}_{(k-1) / 3}\right), \\ \tilde{s}_{k} & \text { otherwise, }\end{cases}
$$$$
\tilde{s}_{k}:=\frac{1}{2} \lambda_{k}\left(\tilde{\mathbf{q}}_{(k-1) / 3}\right)+\frac{1}{2} \lambda_{k}\left(\tilde{\mathbf{q}}_{k / 3}\right) .
$$ 


\begin{tabular}{cccccc}
\hline$\rho_{\mathrm{p}}^{0}$ & $\eta_{\mathrm{p}}$ & $\gamma_{\mathrm{p}}$ & $\rho_{\mathrm{s}}^{0}$ & $\eta_{\mathrm{s}}$ & $\gamma_{\mathrm{s}}$ \\
\hline 1 & 3000 & 7 & $10^{-3}$ & 0 & $7 / 5$ \\
\hline
\end{tabular}

Table 1: Constants in Tait's equation of state (61).

The numerical flux is subsequently computed a $\mathbf{f}_{O M}\left(\mathbf{q}_{L}, \mathbf{q}_{R}\right):=\mathbf{f}(\tilde{\mathbf{q}}(0))$.

Comparison of the approximate Riemann solution (60) with the exact Riemann solution (40) shows that $\tilde{s}_{k}$ acts as an approximation to the shock speed. In Ref. ${ }^{27}$ it is proved that the speed of a shock with strength $\mu$ is equal to the average of the eigenvalues on either side of the shock and a remainder of $O\left(\mu^{2}\right)$, as $\mu \rightarrow 0$.

\section{NUMERICAL EXPERIMENTS AND RESULTS}

To test the non-oscillatory conservative scheme, equipped with the modified Osher scheme, for the numerical fluxes, we consider two test cases. The first test case is a Riemann problem in which the initial velocity and pressure are uniform. Its solution corresponds to a translation of the interface. This test case serves to verify the pressure invariance of the method. The second test case concerns a Riemann problem associated with the collision of a shock with the interface. As a result of the interaction of the shock and the interface, both the conservation properties and the pressure invariance of the method are relevant in this case.

\section{Test CASE I}

We consider the two-fluid Euler equations (9), provided with the compound equation of state (30). The primary and secondary fluid comply with Tait's equation of state (see, e.g., Ref. ${ }^{28}$ ):

$$
\rho_{\mathrm{p} / \mathrm{s}}(p):=\rho_{\mathrm{p} / \mathrm{s}}^{0}\left(\frac{\left(p / p^{0}\right)+\eta_{\mathrm{p} / \mathrm{s}}}{1+\eta_{\mathrm{p} / \mathrm{s}}}\right)^{1 / \gamma_{\mathrm{p} / \mathrm{s}}},
$$

with $p^{0}(:=1)$ an appropriate reference pressure, $\rho_{\mathrm{p} / \mathrm{s}}^{0}$ the corresponding densities of the primary and secondary fluid and $\eta_{\mathrm{p} / \mathrm{s}} \geq 0$ and $\gamma_{\mathrm{p} / \mathrm{s}}>1$ fluid-specific constants. The constants used in the numerical experiments are listed in Table 1. These constants are chosen such that the primary fluid models water and the secondary fluid models air in homentropic flow. Appropriate constants for other fluids are provided in ${ }^{28}$.

Test case I concerns a Riemann problem with

$$
\left(\begin{array}{l}
\rho \\
v \\
g
\end{array}\right)_{0}:=\left(\begin{array}{c}
1 \\
10^{2} \\
1
\end{array}\right) \quad \text { and } \quad\left(\begin{array}{l}
\rho \\
v \\
g
\end{array}\right)_{1}:=\left(\begin{array}{c}
10^{-3} \\
10^{2} \\
0
\end{array}\right) .
$$

So, $p(x, 0)=1$ and $v(x, 0)=100$ for all $x$, i.e., the pressure and velocity are uniform. The solution then corresponds to a translation of the interface.
The two-fluid flow problem is discretized by means of a Godunov-type finite volume method, with the numerical fluxes based on the modified Osher scheme. Instead of a first-order discretization conform (17), we use a limited second order scheme with the minmod limiter (see, e.g., $\left.{ }^{29}\right)$. The intermediate pressure $\tilde{p}_{1 / 2}$ is solved from (54) by means of Newton's method. The integrals in (54) are approximated by 16-point Gauss quadrature. We use a uniform grid with mesh width $h=2^{-6}$. The time step is set to $\tau=2^{-9} h$.

Figure 3 plots the results for test case I. The initial position of the interface is set at $x=0$. The results confirm the pressure invariance of the scheme.

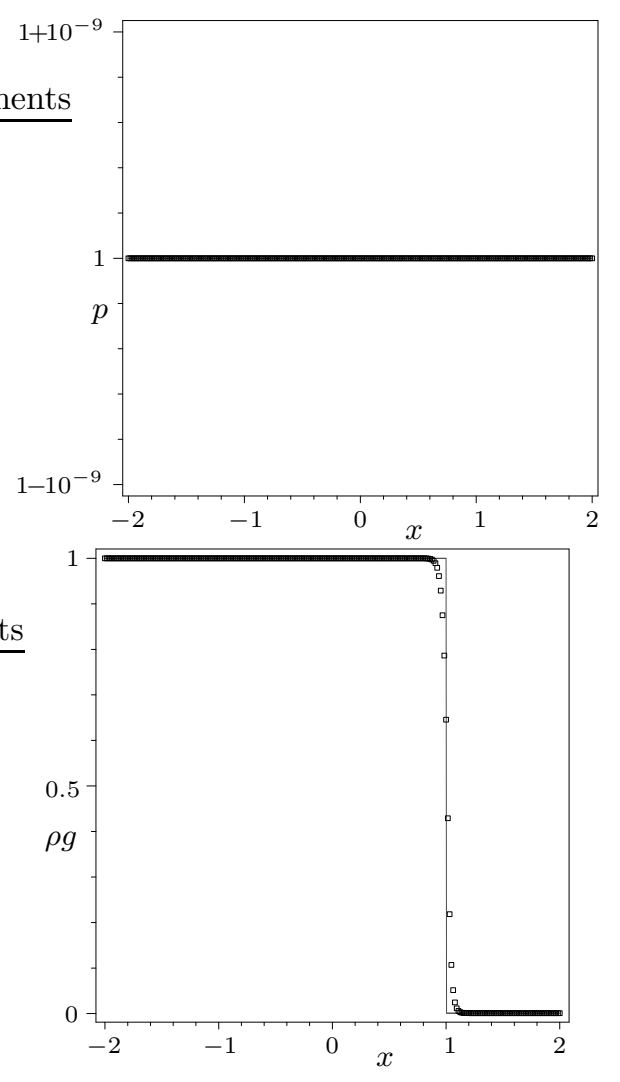

Figure 3: Test case I: Computed result (markers only) and exact solution (solid line)at $t=0.01$. Above: pressure. Below: density.

\section{$\underline{\text { TeSt CASE II }}$}

Test Case II is illustrated in Figure 4. The equation of state of the primary and secondary fluid is specified by (61), with the same constants as in Test Case I (Table 1). The states $\mathbf{q}_{0}, \mathbf{q}_{I}$ and $\mathbf{q}_{1}$ are determined by

$$
\begin{aligned}
\left(\begin{array}{l}
\rho \\
v \\
g
\end{array}\right)_{0}:=\left(\begin{array}{c}
1.000427 \ldots \\
0.062042 \ldots \\
1
\end{array}\right), \quad\left(\begin{array}{l}
\rho \\
v \\
g
\end{array}\right)_{I}:=\left(\begin{array}{l}
1 \\
0 \\
1
\end{array}\right) \text { and } \\
\left(\begin{array}{l}
\rho \\
v \\
g
\end{array}\right)_{1}:=\left(\begin{array}{c}
10^{-3} \\
0 \\
0
\end{array}\right) \cdot \quad
\end{aligned}
$$


The pressure corresponding to $\mathbf{q}_{0}$ is $p_{\mathrm{p}}\left(\rho_{0}\right)=10$. The states $\mathbf{q}_{0}$ and $\mathbf{q}_{I}$ in the primary fluid (water) are connected by a 3 -shock with speed $s_{\mathrm{p}}=145.062002 \ldots$ and $\mathbf{q}_{I}$ is connected to $\mathbf{q}_{1}$ by a steady contact discontinuity, representing the interface. At time $t=0$, the shock collides with the interface, which is set at $x=0$ (see Figure 4). The states $\mathbf{q}_{0}$ and $\mathbf{q}_{1}$ are then contiguous and, hence, the collision induces a Riemann problem. The corresponding Riemann solution assumes the form of a reflected rarefaction wave, a moving interface and a transmitted shock with speed $s_{\mathrm{s}}=37.491063 \ldots\left(=\sigma_{2}^{+}=\sigma_{3}^{-}\right)$.

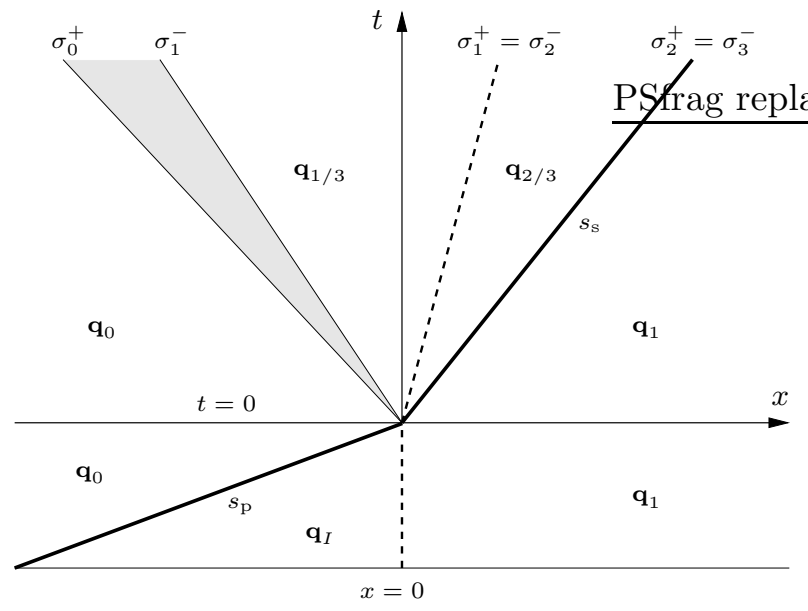

Figure 4: Test case II: The shock/interface collision at $t=$ 0 induces a Riemann problem.

The details of the set up of the numerical experiment for test case II are identical to test case I. In figure 5 we have plotted the results for test case II. The numerical results exhibit good agreement with the exact Riemann solution. We also monitored the mass-conservation errors for the two fluids separately and the momentum-conservation error for this test case: these errors are indeed of the order of the machine precision (results not displayed).

\section{Conclusions}

We presented a non-oscillatory method for barotropic twofluid flows, founded on a formulation of the two-fluid flow problem as a system of hyperbolic conservation laws. The conservative form of the two-fluid flow problem is well suited to treatment by a Godunov-type method. We considered an approximate Riemann solver for barotropic two-fluid flows, based on the rarefaction-waves-only approximation that underlies Osher's scheme.

Numerical results were presented for two Riemann problems, viz., a translating-interface test case and a shock/interface-collision test case. The first test case confirms the pressure invariance of the method. The second test case confirms its conservation properties. In both cases, the computed results agree well with the exact Riemann solution.
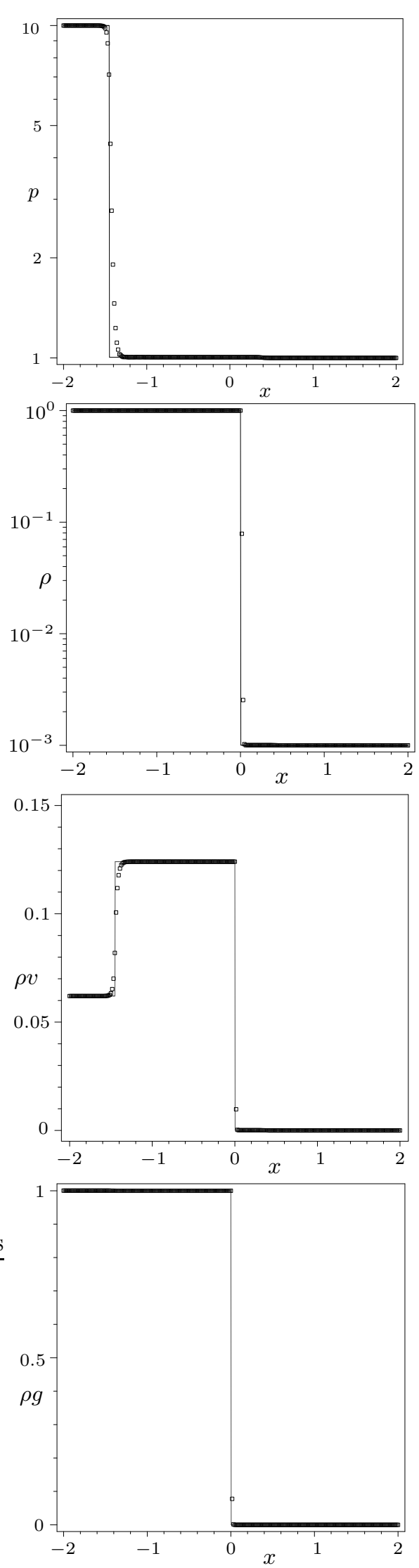

Figure 5: Test case II: Computed result (markers only) and exact solution (solid line) at $t=0.01$. From above to below: pressure (log-scale), density (log-scale), momentum, and primary partial density. 


\section{REFERENCES}

1 R. Abgrall. How to prevent pressure oscillations in multicomponent flow calculations: A quasi conservative approach. J. Comput. Phys. 125, 150-160 (1996).

2 R. Abgrall and S. Karni. Computations of compressible multifluids. J. Comput. Phys. 169, 594-623 (2001).

3 G. Allaire, S. Clerc, and S. Kokh. A five-equation model for the numerical simulation of interfaces in two-phase flows. C. R. Acad. Sci. Paris, Série I. 331, 1017-1022 (2000).

4 E.H. van Brummelen. Numerical Methods for Steady Viscous Free-Surface Flows. CWI Tract 134, CWI, Amsterdam (2003).

5 E.H. van Brummelen and B. Koren. A pressureinvariant conservative Godunov-type method for barotropic two-fluid flows. J. Comput. Phys. 185, 289308 (2003).

6 Y.C. Chang, T.Y. Hou, B. Merriman, and S. Osher. A level set formulation of Eulerian interface capturing methods for incompressible fluid flows. J. Comput. Phys. 124, 449-464 (1996).

7 R.P Fedkiw, T. Aslam, B. Merriman, and S. Osher. A non-oscillatory Eulerian approach to interfaces in multimaterial flows (the ghost-fluid method). J. Comput. Phys. 152, 457-492 (1999).

8 J. Glimm, X.L. Li, Y. Liu, and N. Zhao. Conservative front tracking and level set algorithms. PNAS. 98(25), 14198-14201 (2001).

9 S.K. Godunov. Finite difference method for numerical computation of discontinuous solutions of the equations of fluid dynamics. Mat. Sbornik. 47, 271-306 (1959). (In Russian).

10 P.W. Hemker and S.P. Spekreijse. Multiple grid and Osher's scheme for the efficient solution of the steady Euler equations. Appl. Num. Math. 2, 475-493 (1986).

11 C. Hirsch. Numerical computation of internal and external flows, Volume I: Fundamentals of Numerical Discretization. Numerical Methods in Engineering. Wiley, New York (1995).

12 E. Isaacson and H.B. Keller. Analysis of Numerical Methods. Wiley, New York (1966).

13 P. Jenny, B. Müller, and H. Thomann. Correction of conservative Euler solvers for gas mixtures. J. Comput. Phys. 132, 91-107 (1997).

14 S. Karni. Multicomponent flow calculations by a consistent primitive algorithm. J. Comput. Phys. 112, 3143 (1994).

15 S. Karni. Hybrid multifluid algorithms. SIAM J. Sci. Comput. 17, 1019-1039 (1996).
16 F.J. Kelecy and R.H. Pletcher. The development of a free surface capturing approach for multidimensional free surface flows in closed containers. J. Comput. Phys. 138, 939-980 (1997).

17 B. Koren, M.R. Lewis, Brummelen E.H. van, and Leer B. van. Godunov and level-set approaches for homentropic two-fluid flow computations. J. Comput. Phys. 181, 654-674 (2002).

18 P.D. Lax. Hyperbolic systems of conservation laws II. Comm. Pure Appl. Math. 10, 537-566 (1957).

19 B. van Leer. On the relation between the upwinddifferencing schemes of Godunov, Engquist-Osher and Roe. SIAM J. Sci. Stat. Comput. 5, 1-20 (1984).

20 W. Mulder, S. Osher, and J.A. Sethian. Computing interface motion in compressible gas dynamics. J. Comput. Phys. 100, 209-228 (1992).

21 D. Nguyen, F. Gibou, and R. Fedkiw. A fully conservative ghost-fluid method and stiff detonation waves. In Technical Papers of the 12th International Detonation Symposium. 12th International Detonation Symposium, San Diego, CA (2002). Available at http://www.sainc.com/onr/ det symp/PaperSubmit/FinalManuscript/ pdf/Nguyen-37.pdf.

22 S. Osher and F. Solomon. Upwind difference schemes for hyperbolic conservation laws. Math. Comput. 38, 339-374 (1982).

23 R. Saurel and R. Abgrall. A multiphase Godunov method for compressible multifluid and multiphase flows. J. Comput. Phys. 150, 425-467 (1999).

24 R. Saurel and R. Abgrall. A simple method for compressible multifluid flows. SIAM J. Sci. Comput. 21, 1115-1145 (1999).

25 K. Shyue. A fluid-mixture type algorithm for compressible multicomponent flow with van der Waals equation of state. J. Comput. Phys. 156, 43-88 (1999).

26 K. Shyue. A fluid-mixture type algorithm for compressible multicomponent flow with Mie-Grüneisen equation of state. J. Comput. Phys. 171, 678-707 (2001).

27 J. Smoller. Shock Waves and Reaction-Diffusion Equations. Grundlehren der mathematischen Wissenschaften. Springer, New York (1983).

28 P.A. Thompson. Compressible Fluid Dynamics. Advanced Engineering Series. McGraw-Hill, New York (1972).

29 P. Wesseling. Principles of Computational Fluid Dynamics, volume 29 of Springer Series in Computational Mathematics. Springer, Berlin (2001). 